\title{
AS REPRESENTAÇÕES DOS USUÁRIOS SOBRE A DOENÇA CRÔNICA E A PRÁTICA EDUCATIVA
}

\author{
M aria de Fátima M ANT OVAN Ia, Felismina Rosa Parreira M EN DE S \\ Elis M artins U LBRICH ${ }^{c}$, J anaína M arielen BANDEIRA d, Fernanda F USU M Ad, D aniela M aria GA I ${ }^{d}$
}

\section{RESUMO}

T rata-se de pesquisa qual itativa realizada no período de outubro de 2008 a março de 2009 com 45 usuários maiores de 60 anos de uma unidade de saúde, com o objetivo de identificar as representações dos usuários sobre a doença crônica. A obtenção das representações da doença foi feita por meio de entrevistas e, após foram realizadas três reuniões em grupo, com a média de oito usuários cada. D esta etapa participaram 24 usuários, e os temas oriundos dos discursos foram os seguintes: "A chegada da doença: fatores explicativos", "O mistério das transformações corporais: obrigatoriedade de mudar" e "A importância de aprender e partilhar experiências". Os participantes reconhecem os fatores ligados ao aparecimento da doença e de suas incumbências, as transformações necessárias para continuar vivendo e para postergar complicações valorizam a troca de experiências mediante o diálogo nas atividades educativas.

Descritores: D oença crônica. E ducação em saúde. Promoção da saúde. Cuidados de enfer magem.

\section{RESUMEN}

Se trata de una investigación cualitativa realizada durante el periodo de octubre de 2008 a marzo de 2009, con 45 usuarios mayores de 60 años de una unidad de salud, con el objetivo de comprender las necesidades educativas de las enfermedades crónicas y las percepciones de los usuari os en el mismo. El diagnóstico educati vo fueobtenido por medio de entrevistas. F ueron realizadas tres reuniones en grupo, con un promedio de ocho usuarios cada. D e esta etapa participaron 24 usuarios, y los temas oriundos de los discursos fueron los siguientes: "L a llegada de la enfermedad: factor es explicativos", "E I misterio delas transformaciones corporales: obligatoriedad de mudar" y "L a importancia de aprender y compartir experiencias". L os participantes reconocen los factores ligados al aparecimiento de la enfermedad y de sus incumbencias, las transformaciones necesarias para continuar viviendo y para postergar complicaciones valorizan el intercambio de experiencias mediante el diálogo en las actividades educativas.

Descriptores: E nfermedad crónica. E ducación en salud. Promoción de la salud. A tención de enfermería.

Título: N ecesidades de los ancianos en la enfermedad crónica.

\section{ABSTRACT}

IT his is a qual itative research study held betw een 0 ctober, 2008, and M arch, 2009, with 45 users over 60 years of age from a $\mathrm{H}$ ealth $\mathrm{U}$ nit, which aimed to identify their representations of chronic disease I nerviews w ere car ried out to collect chronic disease's representation. Three team meetings were also carried out, with an average of 8 users. T w enty-four (24) users participated in this step. The themes brought out of their discourses w ere: "T he ar rival of the disease: explanatory factors"; "T hemystery of the bodyly changes: an obligation to change", and "T he importance of learning and sharing the experience". Participants recogniz ed the factors related to the disease onset and their commitments, the changes necessary to keep living and put off complications, and they also valued the exchange of experiences through dialogue in educational activities.

Descriptors: Chronic disease $\mathrm{H}$ ealth education. $\mathrm{H}$ ealth promotion. N ursing care

Title: $U$ ser's representations of chronic diseases and educational practice

\footnotetext{
a D outora em Enfermagem, Professora Associada do D epartamento de Enfermagem da U niversidade Federal do Paraná (UFPR), M embro do Grupo de Estudos M ultiprofissional em Saúde do Adulto (GE M SA-U FPR) e do Centro de I nvestigação em Ciência e T ecnologia da Saúde da U niver sidade de É vora - Portugal, Bol sista Produtividade do Consel ho N acional de D esenvolvimento Científico e T ecnológico (CN Pq), Curitiba, PR, Brasil.

b D outora em Sociologia, Professora Coordenadora da E scola de Enfermagem da U niversidade de É vora, Investigadora do Centro de Investigação e Estudos de Sociologia do Instituto U niversitário de Lisboa (ISCT E-IU L), Lisboa, Portugal.

c M estre em Enfermagem, D outoranda pelo Programa de Pós-G raduação em Enfermagem da UFPR, Bolsista do Programa de A poio a Planos de Reestruturação e Expansão das U niversidades Federais (REUNI), M embro do GEM SA-UFPR, Curitiba, PR, Brasil.

${ }^{d}$ Enfermeira graduada pela UF PR, Curitiba, PR, Brasil.
} 


\section{INT RODUÇÃO}

0 enfermeiro e os demais profissionais de saúde, com a mudança do perfil epidemiológico da população, vem há muito tempo enfrentando problemas referentes à adesão ao tratamento dos portadores de doenças crônicas. Para isto, utilizam a educação em saúde como estratégia na tentativa de modificar os comportamentos e as representações das enfermidades, que se agravam à medida que a população envel hece.

$\mathrm{N}$ os dias atuais, as doenças crônicas são consideradas uma epidemia e um sério problema de saúde pública mundial, sendo estas a principal causa de mortalidade e incapacidade prematura na A mérica $L$ atina e $C$ aribe ${ }^{(1)}$. D entre as enfer midades crônicas destacam-se as cardiovasculares por serem responsáveis por aproximadamente $30 \%$ de todas as mortes no mundo, no qual a Hipertensão A rterial e a Diabetes M ellitus são os principais fatores de risco(2).

Para os profissionais de saúde, o desafio no século XXI é cuidar de uma população de mais de 32 milhões de idosos com nível socioeconômico baixo e com grande número de doenças crônicas(3). Estas constituem patologias dominantes na sociedade atual, com uma prevalência que continua a aumentar, e não se definem somente pelos sintomas físicos e psicológicos, persistindo por toda a vida.

Portanto, a doença acontece entre 0 sujeito e seus mundos sociais, e suas alterações são, na maioria das vezes, mais problemáticas do que a doença, para a qual são prescritos tratamentos ${ }^{(4)}$, cujo objetivo é controlar sua evolução, de modo a prevenir complicações, contribuindo para a diminuição da mortalidade precoce.

0 alcance destes objetivos permitea mel horia da qualidade de vida e da autonomia dos seus portadores se determinados tipos de complicações incapacitantes forem parcial ou totalmente evita$\operatorname{dos}^{(4)}$. N este contexto, a educação em saúde é uma estratégia recomendada para propiciar aos portadores de doença crônica informações pertinentes que os faça compreender e vivenciar o processo da forma mais autônoma possível(5).

Para tanto, utilizam-se metodologias que propiciem a construção/ reconstrução do conhecimento mediantea reflexão do sujeito em inter ação com o seu meio social(5), as quais serão, portanto, válidas se no diagnóstico das atividades educativas propostas pelos profissionais de saúde forem consideradas as crenças em relação à doença, aos hábitos e ao contexto em que vivem os sujeitos alvos da ação(6).

As atividades educativas em grupo permitem o debate e a reflexão e trazem resultados expressivos, pois os participantes sentem-se sujeitos ativos no processo de aprendizagem ${ }^{(7)}$, e podem beneficiar-se com as trocas de experiências que ocorrem entre os membros ${ }^{(5)}$. Essa prática educativa propicia reais condições de aprendizagem entre os educandos.

Tal pensamento norteia a real ização das atividades em busca da promoção da saúde, pois esta apoia o desenvolvimento das pessoas através da informação e da educação em saúde, com vistas à capacitação das pessoas para possibilitar uma vida saudável, de acordo com suas necessidades. 0 intuito é melhorar a qualidade de vida das pessoas com o enfrentamento das condições crônicas de saúde, além de aumentar a capacidade de exercerem controle sobre as suas vidas ${ }^{(8)}$.

Assim, as representações dos educandos acerca da saúde-doença são norteadoras para os profissionais de saúde, pois, em se tratando de percepções da doença, a dupla oposição "saúdedoença" e "indivíduo-sociedade", parte constituinte da mesma, dá sentido a esta e tem a função orientadora de condutas. Essas percepções podem atualizar-se e, se partilhadas, podem coexistir em comportamentos diferentes ${ }^{(9)}$. Por ser "um evento que ameaça ou modifica, às vezes irremediavelmente, nossa vida individual, nossa inserção social e, portanto, o equilíbrio coletivo"(9) a doença produz necessidade de discurso, de interpretação complexa e contínua.

Justifica-se a realização deste estudo tendo em vista a magnitude da doença crônica, a concepção de que a obtenção das representações possibilita aos profissionais identificação de questões do processo de adoecimento muitas vezes pouco conhecidos, principalmente porque embora haja muitos estudos que abordam a temática ainda persistem lacunas neste conhecimento, principalmente se for considerada as diferenças existentes entre os usuários.

Portanto, frente ao exposto, a questão que norteia este trabalho é "quais as representações da doença crônica?". 0 objetivo foi identificar as representações dos usuários sobre a doença crônica. 


\section{METODOLOGIA}

A pesquisa é de natureza qual itativa e foi realizada em uma unidade básica de saúde da Prefeitura M unicipal de Colombo, Região M etropolitana de Curitiba, Paraná. A população em estudo foi constituída por 45 usuários inscritos no Programa de Hipertensão e Diabetes (HIPERDIA), e que compareceram a pelo menos uma das reuniões dos meses de junho, julho ou agosto de 2008.

0 projeto foi aprovado pelo Comitê de Ética em Pesquisa (CE P) do Setor de Ciências da Saúde da U niver sidade Federal do Paraná, em 27 de agosto de 2008, Certificado de A presentação para A preciação É tica (CAAE ) n-2424.0.000.091-08, depois da concordância da Secretaria de Saúde do M unicípio de Colombo.

A coleta de dados foi realizada em duas etapas: a primeira uma entrevista domiciliar com duração média de duas horas, após esclarecimentos e a assinatura do Termo de Consentimento Livre e Esclarecido (T CLE), a qual permitiu a identificação das necessidades educativas dos usuários para as reuniões em grupo, que constituíram a segunda etapa. D os 45 entrevistados 33 aceitaram participar das reuniões de grupo e foram convidados por tel efone, destes 24 comparecer am a pel o menos uma reunião. Foram realizados três encontros grupais, com duração de uma hora e meia, com a média de oito participantes da comunidade e cinco acadêmicos do curso de Enfermagem, sendo um deles 0 mediador, e os demais real izavam as tar efas de gravação e auxílio nas atividades educativas.

As reuniões de grupo foram assim organizadas: durante os cinco minutos iniciais era feita uma apresentação dos objetivos de cada encontro e das dinâmicas que seriam utilizadas na apresentação de todos os participantes. N o tempo restante só os novos integrantes se apresentavam, e todos diziam os seus nomes, com sessenta minutos para a discussão e síntese do tema e trinta minutos finais para avaliação e convivência.

Os depoimentos oriundos das reuniões foram transcritos para compor o corpus de conhecimento que foi analisado mediante a técnica de análise de conteúdo, e os participantes foram identificados com a letra $S$ seguida de um número. Foram obtidas três categorias temáticas: "A chegada da doença: fatores explicativos", "O mistério das transformações corpor ais: obrigatoriedade de mudar" e "A importância de aprender e partilhar experiências".

\section{RESULTADOS E DISCUSSÃO}

Os participantes das reuniões de grupo são 17 mulheres e 7 homens com idade variando entre 61 e 94 anos; 9 deles vivem com um companheiro/ cônjuge, 12 são viúvos e 3 separados ou divorciados; 15 possuem o ensino fundamental incompleto, 8 não eram alfabetizados e 1 concluiu o ensino médio. Em relação à profissão/ ocupação, 2 possuem atividade remunerada, 15 são aposentados ou pensionistas da seguridade social e 7 são do lar. O bserva-se que este perfil é similar ao apresentado em estudos com amostras oriundas de programas de saúde como este, e a diferença encontrase na alta concentração de não alfabetizados ${ }^{(10)}$, fato comum entre pessoas nesta faixa etária ${ }^{(11)}$.

0 tempo de diagnóstico das doenças hipertensão e diabetes variou de 6 meses a 47 anos; a maioria dos participantes tem hipertensão arterial, 8 possuem diabetes e hipertensão e um deles possui apenas diabetes. Todos utilizam a unidade de saúde para tratamento e aquisição dos medicamentos ou os retiram em outras unidades do município. Concomitantemente ao tratamento medicamentoso, alguns usuários ingerem vários tipos de chás de ervas, como chá de hortelã, erva cidreira, capim limão, carqueja e o chimarrão.

A utilização de ervas medicinais como auxiliar do tratamento propicia, de certa forma, um controle destes usuários sobre o seu cotidiano modificado pela doença ${ }^{(4)}$, pois cada indivíduo, com base em suas experiências pessoais, manifesta suas concepções do processo de adoecimento e tratamento, as quais mantêm relações com suas crenças, pensamentos e sentimentos. Não há uma preocupação com a cientificidade na utilização de tratamentos al ternativos, nem mesmo na certeza da efetividade de suas ações(12).

Ao serem questionados na entrevista sobre 0 que "consideravam mais difícil de entender sobre a sua doença", direcionaram as suas respostas à mudança de hábitos de vida, aos aspectos fisiopatológicos da doença e suas complicações, à adesão ao tratamento e à aceitação da cronicidade por eles e pelos membros da família, como nos depoimentos a seguir:

Por que isto? Por que dá diabetes? D e onde vem a doença e por quê? (S 15).

[ ...] minha família não entende a doença (S 2). 
A ceitar a doença, não sei explicar o que gostaria de saber (S 1).

D eixar de comer doce; por que os sintomas aparecem? (S 31).

Como fazer para melhorar a diabetes e a hipertensão arterial? (S 43).

Nesses trechos observa-se que as dúvidas apresentadas dão a conotação de que essas pessoas nunca foram informadas acerca da doença, do tratamento, dos fatores de risco, entre outros aspectos. Estas necessidades educativas identificadas nas entrevistas serviram de temas para os encontros em grupo: definição de hipertensão e diabetes, fatores de risco, as complicações das doenças, o tratamento e a cronicidade.

Os assuntos requeridos são similares aos abordados pelos profissionais de saúde quando trabaIham as questões do diabetes e/ ou da hipertensão em grupos ou individualmente. E m uma pesquisa bibliog ráfica real izada sobre educação em saúde e hipertensão(13), verificou-se que os temas das atividades educativas, em sua maioria, referiam-se ao tratamento, ao controle dos fatores de risco eà prescrição de cuidados, o mesmo ocorrendo com a atividade aqui realizada.

Os conteúdos dos depoimentos dos usuários nas reuniões de grupo propiciaram a construção de três categorias; a primeira "A chegada da doença: fatores explicativos" verificou-se que eles não entendiam o caráter crônico da mesma, pois descreveram seu aparecimento aliado a complicações e narraram formas utilizadas para contor nar a sua aparição, como apontado no trecho a seguir:

\begin{abstract}
$M$ as teve uma época, quefaz uns cinco ou seis anos mas não sofria de pressão, nada. $N$ ão tinha problema ne nhum [ ...] eu era teimoso, não gostava deir no médico. [ ...] Ali no ponto tinha um farmacêutico, eu fui medir a pressão. E stava 18×20, ele disse, mas está aguentando? E u melhorei daí, eu estou bom, não estou sentindo nada enão senti nada lá também. T inha uma mercearia, eu vou tomar uma dose de conhaque para curar de vez [ ...] tomei um conhaque [ ...] Ai continuei assim, daí fui medindo a pressão, daí eu comprei um remédio lá e não deu certo, enão consegui tomar mais. N o mês dejulho, me deu um começo de der rame [...] (S 1).
\end{abstract}

Observa-se que a procura por recursos de saúde ocorreu no momento da agudização de alguns sintomas, e finalmente, após um início irresoluto com um tratamento mal feito, houve "um começo de derrame", que, sendo um fato novo, incentivou a procura do tratamento. A busca por alternativas antes do diagnóstico é referida na literatura, pois as condutas iniciais antes de procurar um médico são de apelar para a medicina caseira ou religiosa ou mesmo para o "farmacêutico", e só mesmo com o ag ravamento da situação é que há a exigência de um tratamento com profissional de saúde ${ }^{(12)}$.

A qui também pode se observar que pela falta de sintomatologia surge a negação do adoecimento, que também é fator que dificulta não só o início como a continuidade do tratamento ${ }^{(4,10)}$. "Os doentes fazem sempre escolhas face às pressões da doença e do tratamento", pois estes nem sempre são aceitos de imediato, sendo avaliados em ter mos de consequências e alterações no cotidiano, fatores muito mais decisivos que os critérios clínicos ${ }^{(4)}$.

Outro aspecto desta categoria são os fatores explicativos para a chegada da doença como 0 stress, a correria do dia-a-dia e as alter ações provocadas pelo ner vosismo.

Sistema nervoso, nervoso, todo dia, todo dia, até que leva a pressão a subir por causa do nervoso (S 7).

Verificou-se que há similaridade destes achados com outros estudos relativos à representação da hipertensão ${ }^{(10,14)}$, nos quais a doença foi associada a questões emocionais, à modificação da consistência do sangue, a hábitos de vida inadequados para a saúde, principalmente a dieta.

A categoria "O mistério das transformações corporais: obrigatoriedade de mudar" expressa 0 diálogo ocorrido nas reuniões em grupo, apesar dos assuntos discutidos durante os encontros serem oriundos das entrevistas realizadas nos domicílios, o cerne da dúvida são as questões intrínsecas da fisiologia do corpo que contêm as alterações, não podem ser visualizados. Ou seja, procuram-se respostas para questões como "por que mudou?" e "por que não volta ao normal?".

Contribuíram [ ...] mas eu ainda tenho dúvida no que ocorre no organismo para a pressão subir (S 24).

Este depoimento chama a atenção dos profissionais de saúde, pois a multicausalidade associada à doença crônica remete os indivíduos para a incer teza e os profissionais para a incapacidade de elucidarem sobre o que desencadeou a doença. 
Social izados na crença de que a medicina identifica a causa e cura todos os males, face à doença crônica essas exigências mantêm-se quando tal crença não é satisfeita. As seguintes indagações são relevantes: por que a doença surgiu, por que não se cura e como vai evoluir? A identificação causal da doença cria importantes pontos de referência na interface do eu com a sociedade e é igualmente importante para ajudar a interpretar diferentes aspectos da biografia de cada um, para realinhar o presente com 0 passado e para poder conjugar o presente no futu$\mathrm{ro}^{(15)}$.

E m relação à necessidade de mudar para continuar vivendo, os usuários abordam aspectos da dieta, do abandono de vícios e até da necessidade de se cuidar para não morrer:

E u parei de trabalhar, então aí eu não sabia nada de pressão. $L$ arguei de tudo quanto é tipo de vício. [ ...] Como eu disse, você morre e só uma ponte de safena te salva. I sso foi nos fins dos anos de 79, edaí eu fui aposentado, e dali eu comecei a fazer tratamento até hoje (S 23).

E u não gosto muito de comida muito sem sal, mas diminuí também, não salgada, mas fui diminuindo um pouco. E ntão a gente procura não deixar defazer, não sei, eu posso estar até er rado, mais também não fazer tudo aquilo que eu fazia antes (S 3).

A gente tem que levar a sério os remédios! A gente não quer mor rer logo (S 8).

N ota-se que os usuários reproduzem o discurso dos profissionais de saúde em relação às mudanças necessárias para o controle das doenças e falam da morte, que é uma possibilidade que advém do descontrole e das complicações, mas 0 medo de morrer pode incentivar a adesão às orientações for necidas pela equipe de saúde. 0 discurso da saúde tem conquistado um público crescente, e os doentes crônicos que vivem na eminência de episódios de agudização parecem emergir como destinatários privilegiados desta ideologia ${ }^{(15)}$.

As mudanças exigidas para continuar vivendo referem-se aos hábitos e estilos de vida, o uso de medicamentos prescritos, o controle da dieta, 0 abandono dos vícios, permitindo a estes portadores de doença crônica manterem-se controlados. Percebe-se, portanto, que pelo menos momentaneamente as questões que eram dúvidas e dificuldades de entendimento sobre a doença durante as entrevistas, nas reuniões em grupo foram sanadas, mas sabe-se que as mudanças de comportamento resultantes das atividades educativas, se ocorrerem, fazem parte de um processo complexo "que inclui fatores subjetivos e objetivos, como, por exemplo, o contexto social, econômico e cultural, bem como as motivações individuais"(5), pois uma das facetas do doente crônico é a de gerir o seu tratamento.

Os doentes crônicos gerem as consequências da doença na sua vida, na tentativa de manter 0 seu papel de intervir como elemento principal do seu tratamento. 0 doente utiliza a sua capacidade defazer escolhas eatender aquelas que lhe aprouver, negociando com seu mal-estar e ajustando-se emocionalmente às suas escolhas ${ }^{(4)}$.

Os depoimentos destes usuários corroboram com outros estudos sobre a temática que também enfatizam, o controle de fatores estressantes, o lazer e a tranquilidade de vida. Estes aspectos ligam-se à aprendizagem que a doença impõe aos doentes. Eles não são apenas doentes, eles aprendem a ser doentes. Simultaneamente, a doença dá-Ihes a ocasião para repensarem a sua vida (passada e presente) e legitima as mudanças que acionam para obterem o controle sintomático ${ }^{(16)}$.

0 uso correto da medicação é abordado como uma necessidade para postergar a morte e continuar vivendo sem complicações ${ }^{(14)}$. N o entanto, esta adoção do tratamento pelo doente nem sempre lhe garante melhora dos sintomas da doença, e reside aí um grande problema, difícil de ser aceito pelos doentes: por mais que façam, não Ihes é garantido nem a mel hora da doença nem que não haverá complicações da mesma. A incerteza, como um marco decisivo da doença crônica, está mais uma vez presente nos testemunhos recol hidos.

A morte aparece da mesma forma que em outros estudos, como uma possibilidade presente no adoecimento, em que a falta de adesão ao tratamento pode trazê-la rapidamente. A morte é uma perspectiva inexorável presente em muitos discursos dos participantes. Para eles, ou há aceitação e seguimento do tratamento, ou a vida é encurtada em alguns anos. A presença do Divino na manutenção da vida também foi lembrada, e a Ele são atribuídas a melhora ou mesmo as intercorrências do adoecimento, além de, às vezes, até a cura das situações crônicas de saúde $e^{(10,11)}$

"A importância de aprender e partilhar experiências" é a terceira categoria e surgiu na avalia- 
ção das atividades educativas nas reuniões de grupo, pois estas possibilitaram um espaço livre de troca de informações, expresso abaixo pelo Sujeito 7, indo ao encontro do preconizado neste tipo de abordagem, pois ao expressarem suas idéias possibilitam mel hores condições de cuidado de si e dos ou$\operatorname{tros}^{(7)}$

M as é por que nós estamos aqui? 0 que nós estamos faz endo aqui? A prendendo. $N$ ão é verdade? $\mathrm{N}$ ós estamos aqui aprendendo. A gora qualquer um de nós com uma pequena palavra podetrazer grandeproveito para 0 outro. Às vezes até um pensamento (S 7).

P orque a gente hoje em dia tem que aprender também pra viver um pouco mais, porque a minha família, todos eles mor reram cedo também, meu pai e minha mãe de pressão alta e diabetes, porque naquela época não tinha assim reuniões e também no sítio eles não entendiam nada. E ntão, meu marido não é muito assim, ele não quer nem participar [ ...] edepois que eu comecei a vir nas reuniões e tomar remédio da pressão eu faço caminhada efaço ex ercício, nossa, mesinto uma menina (S 3).

Pode-se perceber que há uma preocupação em partilhar as experiências pessoais, e que estas influenciam e colaboram com o aprender do outro, pois cada um, baseado no que viveu em relação à doença, manifesta as suas concepções do processo de adoecimento de acordo com suas crenças ${ }^{(10)}$, esta partilha de experiência ${ }^{(16)}$, tão característica dos doentes crônicos, visa romper com a solidão e o isolamento e está no centro das motivações daqueles que procuram os grupos de autoajuda. Neles encontram o apoio, um discurso semel hante sobre a doença, os seus sintomas e as crises de agudização e aprendem a cuidar diariamente de sua doença. Esta troca de experiências permite ainda aos doentes perceberem que o seu caso não éúnico e, assim, encontram pontos de referência para organizarem as suas atividades de compreensão e controle da sua patologia.

Neste estudo, a participação foi valorizada pelos usuários, que assinalam que as questões discutidas ajudam a viver mais e mel hor, pois com "a efetiva participação comunitária é possível assegurar sustentabilidade e efetividade das ações de saú$\mathrm{de}^{\prime \prime(13)}$, além de incentivar a autonomia dos sujeitos "reconhecendo-os como portadores de um saber que, embora diverso do saber técnico-científico, não deve ser deslegitimado pelos serviços"(13), corro- borando, portanto, com a perspectiva da promoção da saúde, que preconiza a participação ativa dos sujeitos e a sua corresponsabilização em assuntos de saúde.

\section{CONSIDERAÇÕES FINAIS}

As necessidades apontadas pel o grupo em relação à doença crônica são as normalmente identificadas como importantes pelos profissionais de saúde, cujo discurso é o de respeitar o contexto e a subjetividade dos seus portadores. Temos que refletir até que ponto as dificuldades sentidas por eles não são, de alguma maneira, induzidas por aquilo que constantemente insiste-se em abordar. Se o que eles necessitam é que se continue a falar da doença, é dela que deve ser falado, até que se esgotem as dúvidas e que haja minimamente compreensão do processo de adoecimento crônico. I sto significa que os profissionais têm que encarar os doentes crônicos como seus parceiros na produção e informação de cuidados, e não como meros destinatários ou receptores dos mesmos.

Significa também que os saberes que o doente vai adquirindo, ao longo do tempo, vão sendo apropriados e destinam-se a satisfazer as necessidades inerentes a cada fase da doença. N este sentido, é sempre o doente e a sua necessidade que comanda o processo de aquisição de informação, e não o profissional, porque nem todos os doentes experimentam da mesma forma o processo através do qual têm que aprender a "ser doentes" (ao contrário da doença aguda em que "estavam doentes").

As categorias de representação das necessidades demonstram que, apesar de toda mídia em torno da prevenção dos fatores de risco para os problemas cardiovasculares, há lacunas muito importantes no conhecimento, as quais podem estar relacionadas com o nível de instrução dos participantes ou mesmo com a aceitação da condição crônica.

E $m$ relação à atividade educativa de enfermagem considera-se que é necessário continuar buscando diagnósticos, representações de processos de adoecimento no contexto em que a população alvo da educação em saúde esteja inserida, e manter o diálogo, pois ele propicia a troca de experiências extremamente importante na promoção da saúde dos portadores de doença crônica. 


\section{REFERÊNCIAS}

1 M inistério da Saúde, Secretaria de Vigilância à Saúde, Secretaria de A tenção à Saúde. Diretrizes e recomendações para o cuidado integral de doenças crônicas não-transmissíveis: promoção da saúde, vigilância, prevenção e assistência. Brasília (D F); 2008.

2 Sociedade Brasileira de Hipertensão, Sociedade Brasileir a de Cardiologia, Sociedade Brasileira de N efrologia. V Diretrizes Brasileiras de Hipertensão Arterial. São Paulo; 2006.

3 Ramos L R. F atores deter minantes do envelhecimento saudável em idosos residentes em centro urbano: Projeto E pidoso, São Paulo. Cad. Saúde Pública. 2003; 19(3):793-8.

4 M endes FR P. D oenças crônicas: a prioridade de gerir a doença e negociar os cuidados. Pensar Enferm. 2005;9(1):42-7.

5 Souza AC, Colomé ICS, Costa LED, Oliveira DLLC. A educação em saúde com grupos na comunidade: uma estratégia facilitadora da promoção da saúde. Rev $\mathrm{G}$ aúcha Enferm. 2005;26(2):147-53.

6 Pires CGS, M ussi FC. Refletindo sobre pressupostos para o cuidar cuidado na educação em saúde para a pessoa hipertensa. Rev Esc Enferm USP [ Internet] . 2009 [ citado 2009 jun 10];43(1):229-36. Disponível em: http:/ / www.scielo.br/ pdf/ reeusp/ v43n1/ 30. pdf.

7 Severo I, Silva M , G orini M . Analysis about production of nursing knowledge on health education and aging: review article. Online Braz J Nurs [Internet] . 2008[ cited 2009 Jan 14] ;7(1). Available from: http:/ / www.uff.br/ objnursing/index.php/ nursing/article/ view/ 1252.

8 Budó M LD, M attioni FC, M achado TS, Ressel LB, Lopes LFD. Quality of life and health promotion through the perspective of the users of the family health strategy. Online Braz ] N urs [ I nter net] . 2008 [ cited 2009 ] an 06] ;7(1). Avail able from: http:/ / www.

\section{Endereço da autora / Dirección del autor / Author's address:}

$M$ aria de $F$ átima $M$ antovani

Av. Lothário M eissner, 632, 3o andar, Jardim Botânico 80210-170, Curitiba, PR

E-mail: mfatimamantovani@ufpr.br uff.br/ objnursing/ index.php/ nursing/ article/ view/ 1104.

$9 \mathrm{H}$ erzlich C. A problemática da representação social e sua utilidade no campo da doença. Physis. 2005;15 (Supl):57-70.

$10 \mathrm{M}$ antovani M F, U Ibrich EM , Pinotti S, G iacomozzi LM , Labronici L M, Sarquis L M. 0 significado e a representação da doença crônica: conhecimento do portador de hipertensão acerca de sua enfermidade. Cogitare E nferm. 2008;13(3):336-42.

11 Andrade OG. Representações sociais da saúde e da doença na velhice. Acta Sci, H ealth Sci. 2003;25(2): 207-13.

12 Siqueira K M , Barbosa M A, Brasif VV, Oliveira L M C, Andraus L M S. Crenças populares referentes à saúde: apropriação de saberes sócio-culturais. T exto Contexto Enferm. 2006;15(1):68-73.

13 Toledo M M, Rodrigues SC, Chiesa A M . Educação em saúde no enfrentamento da hipertensão arterial: uma nova ótica para um velho problema. Texto Contexto Enferm. 2007;16(2):233-8.

14 Péres DS, M agna J M , Viana LA. Portador de hipertensão arterial: atitudes, crenças, percepções, pensamentos e práticas. Rev Saúde Pública [ Internet] . 2003 [ citado 2009 jan 15] ;37(5):635-42. Disponível em: http:// www.scielosp.org/ pdf/ rsp/v37n5/ 17480.pdf.

15 W illiams $G$. T he genesis of chronic illness. In: Bury M, G abe J. The sociology of health and illness: a reader. L ondon: Routledge; 2004. p. 247-55.

$16 \mathrm{H}$ erzlich C, Pierret J. M alades d'hier, maladies d'aujourd'hui. Paris: Payot; 1991.

\section{AGRADECIMENTOS}

À Coordenação de A perfeiçoamento de Pessoal de $N$ ível Superior (CAPES) pelo financiamento com a bolsa de pós-doutoramento.

Recebido em: 22/ 11/ 2010

A provado em: 21/ 11/ 2011 